\title{
Leitos hospitalares e reforma psiquiátrica no Brasil
}

\author{
Hospital beds and mental health reform in Brazil
}

\footnotetext{
${ }^{1}$ Laboratório de Economia Social, São Paulo, Brasil.

2 Secretaria de Economia e Planejamento do Estado de São Paulo, São Paulo, Brasil.

Correspondência

S. Kilsztajn

Laboratório de Economia

Social.

Rua Marquês de Paranaguá 164, apto. 602, São Paulo, SP 01303-050, Brasil.

skilmail@gmail.com
}

\section{Abstract}

The objective of this study was to estimate the number of psychiatric beds occupied per State in Brazil and the amount paid by the Unified $\mathrm{Na}$ tional Health System (SUS) for hospitalizations, professional services, tests, and medicines in the country in 2004. The mean number of psychiatric beds occupied, estimated on the basis of total days of hospitalization during the year, and the amount paid by the SUS were obtained from the Hospital Admissions Authorizations (AIH). A total of 45 thousand psychiatric beds were occupied by the SUS in 2004. The SUS paid a total of BRL $\$ 487$ million (some U\$270 million) for hospitalization of patients with mental disorders in 2004. Private hospitals accounted for $78.8 \%$ of all psychiatric beds occupied by the SUS. Although the deactivation of 15 mil psychiatric beds could currently generate BRL $\$ 162$ million (U\$90 million) to be reallocated to non-hospital psychiatric services, planning and implementation of the Psychiatric Reform have been very limited. The precarious extra-hospital network has been used as a barrier to deactivation of psychiatric beds, although the latter generates the necessary resources for the former

Hospital Bed Capacity; Psychiatric Hospitals; Mental Disorders

\author{
Samuel Kilsztajn 1 \\ Erika de Souza Lopes 1 \\ Luciana Zilles Lima 1 \\ Patrícia Avanzini Ferreira da Rocha 1 \\ Manuela Santos Nunes do Carmo 1,2
}

\section{Introdução}

A adequação dos hospitais psiquiátricos e seus métodos terapêuticos foram radicalmente questionados na década de 1960 por Franco Basaglia; e a Itália, em 1978, foi o primeiro país do mundo a aprovar uma lei antimanicomial 1 . No Brasil, a psiquiatra Nise da Silveira destacou-se por sua batalha contra os tratamentos à base de eletrochoques, lobotomia e camisas-de-força químicas; pela utilização da pintura e da modelagem como ocupação terapêutica e de animais para relações "pessoais" com esquizofrênicos; e pela criação, em 1956, da Casa das Palmeiras, o centro de atenção psicossocial em regime de externato com portas e janelas sempre abertas 2 .

O movimento pela desinstitucionalização dos pacientes que apresentam transtornos mentais desenvolveu-se no Brasil na segunda metade da década de 1980 e gerou, em 1989, o Projeto de Lei $n^{o}$. 3.657 de Paulo Delgado 3. A Reforma Psiquiátrica, que dispõe sobre a proteção e os direitos das pessoas portadoras de transtornos mentais e redireciona o modelo assistencial em saúde mental, contudo, só foi decretada pelo Congresso Nacional e sancionada pela Lei $n^{o}$. $10.216 \mathrm{em}$ 2001 4. Em 2005, o Tribunal de Contas da União publicou o relatório Avaliação das Ações de Atenção à Saúde Mental: Programa Atenção à Saúde de Populações Estratégicas e em Situações Especiais de Agravos 5 com várias críticas à morosidade do processo de desospitalização em curso. 
De acordo com o relatório 5, os hospitais psiquiátricos no Brasil, tradicionalmente, eram (e continuam sendo) responsáveis tanto pela residência como pelo atendimento terapêutico a pacientes que apresentam transtornos mentais. A redução progressiva de leitos e reinserção social dos pacientes psiquiátricos devem ser, desta forma, acompanhadas, por um lado, pela reintegração dos internos aos lares de seus familiares ou pela criação de residências terapêuticas (Programa De Volta para Casa, Serviços Residenciais Terapêuticos - SRT); e, por outro, pela expansão dos Centros de Atenção Psicossocial (CAPS), pela inclusão de ações de saúde mental na atenção básica, Programa Saúde da Família (PSF) e outros serviços de saúde, e pelo credenciamento de leitos psiquiátricos em hospitais gerais para situações singulares em que a internação se faça necessária.

Para contribuir com a discussão da Reforma Psiquiátrica e reintegração social dos internos, o objetivo deste trabalho é estimar o número de leitos psiquiátricos ocupados por Unidade da Federação e a magnitude do valor pago pelo Sistema Único de Saúde (SUS) nas internações por transtornos mentais por serviços hospitalares, serviços profissionais, exames e medicamentos no Brasil. Para tornar compatível esta pesquisa com o dados levantados pelo Tribunal de Contas da União 5 optou-se por limitar o trabalho ao ano de 2004.

\section{Métodos}

O número de leitos psiquiátricos ocupados pelo SUS foi estimado a partir dos dias de permanência registrados nas Autorizações de Internação Hospitalar (AIH) 6 e comparado ao número de leitos psiquiátricos cadastrados pelo SUS 7 e ao número de leitos/SUS registrados no Cadastro Nacional de Estabelecimentos de Saúde (CNES) 8 .

Os dias de permanência nas AIH correspondem ao total de dias de internação no mês de competência (mês imediatamente anterior ao da apresentação das AIH para faturamento). As AIH, contudo, podem incluir períodos de internação anteriores ao mês de competência (AIH apresentadas com atraso ou reapresentadas por terem sido rejeitadas em alguma competência anterior). Mas, em nível agregado, eventuais atrasos são desprezíveis e, para séries anuais, as defasagens em meses específicos compensam-se ao longo do ano. Para o exercício de 2004, com o objetivo de confirmar a correção deste procedimento, os dias de permanência foram conferidos por hospital e mês de competência ${ }^{9}$.
Os dias de permanência, formalmente, incluem períodos em que o paciente utiliza UTI e, portanto, não utiliza leito. Entretanto, entre os pacientes internados por transtornos mentais e comportamentais (capítulo V da Classificação Internacional de Doenças, 10a Revisão - CID-10 10), a utilização de UTI é muito pouco expressiva. Os dias de UTI correspondiam a $0,005 \%$ do total de dias de permanência de pacientes internados por transtornos mentais em 20049.

O número médio de leitos psiquiátricos ocupados foi estimado a partir do total de dias de permanência no ano dividido pelo número de dias no ano. Para o número de leitos psiquiátricos ocupados por mil habitantes entre $1994 \mathrm{e}$ 2004 foram utilizados os dias de internação 6 e as estimativas da população residente 11. Para 2004, foram também calculados os percentuais de leitos psiquiátricos no total de leitos ocupados pelo SUS por Unidade da Federação ${ }^{6}$.

Para a distribuição dos leitos psiquiátricos ocupados por especialidade foram utilizados os dias de internação por grupos da CID-10 $10 \mathrm{em}$ 20049 . Foram calculados ainda, por especialidade, o número e percentual de leitos psiquiátricos ocupados em dezembro de 2004 (dias de internação no mês divididos por 31) por pacientes com data de internação anterior a julho do mesmo ano.

O valor total pago pelo SUS relativo a internações de pacientes com transtornos mentais em 2004, dividido em serviços hospitalares, profissionais e SADT (serviços auxiliares de diagnose e terapia: exames e medicamentos), também foi obtido a partir das AIH 9. O valor pago por paciente/mês em 2004 foi calculado a partir do valor anual dividido pelo número total de dias de permanência, multiplicado por 365/12. Foi calculado também o volume de recursos das AIH a serem gerados com a desativação de leitos de pacientes asilares potencialmente beneficiários de desinstitucionalização, de acordo com as estimativas do Ministério da Saúde 5 .

Para a distribuição dos hospitais por número médio de leitos psiquiátricos ocupados foram consideradas quatro categorias: até 40; 41-120; 121-200; e mais de 200 leitos por hospital. O número de leitos, hospitais e o valor pago pelo SUS para as internações por transtornos mentais foram distribuídos de acordo com a natureza dos hospitais: privados (contratados e filantrópicos), federais, estaduais e municipais.

\section{Resultados}

De acordo com a Tabela 1, o número de leitos psiquiátricos cadastrados pelo SUS 7 durante 
todo o período de análise é sistematicamente superior (entre $27 \%$ e $41 \%$ ) à estimativa de leitos psiquiátricos efetivamente ocupados pelo SUS. O CNES 8, por sua vez, registrava 47.911 leitos psiquiátricos em 4 de agosto de 2005, número $12 \%$ superior à estimativa de 42.695 leitos psiquiátricos ocupados em julho de 20056 .

Tanto o número de leitos cadastrados como as estimativas de leitos ocupados por mil habitantes apresentaram queda no período analisado. A taxa por mil habitantes registrou 0,36 para leitos cadastrados em julho de 2003 e 0,25 para leitos ocupados em 2004.

Em números absolutos, os estados com maior número de leitos psiquiátricos ocupados em 2004 eram São Paulo (14.189 leitos), Rio de Janeiro (7.943), Paraná (3.488), Pernambuco (3.326) e Minas Gerais (3.191). A Figura 1 apresenta as taxas de leitos psiquiátricos ocupados por mil habitantes em 2004, que eram expressivamente diferentes entre as Unidades da Federação. Rio de Janeiro, Pernambuco, São Paulo, Paraná, Alagoas, Rio Grande do Norte e Paraíba apresentaram taxas superiores à média nacional $(0,25)$; e os estados da Região Norte apresentaram baixas taxas de hospitalização de pacientes com transtornos mentais. A Figura 1 também apresenta os percentuais de leitos psiquiátricos no total de leitos ocupados pelo SUS por Unidade da Federação. Via de regra, os estados com maiores taxas de leitos psiquiátri- cos ocupados por mil habitantes apresentaram também maiores percentuais de leitos psiquiátricos no total de leitos ocupados pelo SUS.

Os pacientes classificados no grupo esquizofrenia, transtornos esquizotípicos e delirantes ocuparam $54,4 \%$ do total de leitos psiquiátricos. Os pacientes classificados nos grupos transtornos mentais devidos ao uso de substâncias psicoativas (particularmente álcool); transtornos mentais orgânicos, inclusive sintomáticos (demência etc.); transtornos de humor - afetivos (depressão etc.); e retardo mental ocuparam respectivamente $14,7 \%, 13,9 \%, 8 \%$ e $7,9 \%$ do total de leitos psiquiátricos. Os demais cinco grupos e os transtornos mentais não especificados, somados, ocuparam $1,1 \%$ do total de leitos psiquiátricos em 2004 (Tabela 2).

Em dezembro de 2004, o total de leitos psiquiátricos ocupados por pacientes com data de internação anterior a julho de 2004 era de 17.885, correspondendo a $41,1 \%$ do total de leitos psiquiátricos ocupados. Os pacientes com data de internação anterior a julho de 2004 pertenciam essencialmente aos grupos esquizofrenia, transtornos orgânicos e retardo mental.

O valor total pago pelo SUS para internações de pacientes com transtornos mentais atingiu R\$ 487 milhões em 2004. O valor médio por paciente/mês foi calculado em R\$749,12 para serviços hospitalares, $\mathrm{R} \$ 75,08$ para serviços

Tabela 1

Leitos psiquiátricos/Sistema Único de Saúde (SUS) por mil habitantes. Brasil.

\begin{tabular}{lccccc}
\hline Ano & Cadastrados * & Ocupados ** & $\begin{array}{c}\text { Cadastrados/ } \\
\text { Ocupados }\end{array}$ & $\begin{array}{c}\text { Leitos por mil habitantes } \\
\text { Cadastrados }\end{array}$ \\
\hline 1994 & 87.134 & 61.943 & 1,41 & 0,57 & 0,40 \\
1995 & 83.380 & 59.014 & 1,41 & 0,54 & 0,38 \\
1996 & 76.999 & 56.725 & 1,36 & 0,49 & 0,36 \\
1997 & 76.515 & 55.958 & 1,37 & 0,48 & 0,35 \\
1998 & 71.848 & 56.031 & 1,28 & 0,44 & 0,35 \\
1999 & 73.149 & 54.787 & 1,34 & 0,45 & 0,33 \\
2000 & 71.074 & 54.632 & 1,30 & 0,42 & 0,32 \\
2002 & 69.394 & 52.901 & 1,31 & 0,40 & 0,31 \\
2003 & 64.736 & 51.163 & 1,27 & 0,37 & 0,29 \\
\hline
\end{tabular}

Fonte: Departamento de Informática do SUS 6,7,11.

Nota: 47.911 leitos SUS e 8.672 não SUS (total 56.583) em 04 de agosto de 2005 de acordo com Cadastro Nacional de Estabelecimentos de Saúde 8 .

* Leitos cadastrados em julho;

** Leitos ocupados (média anual): dias de permanência divididos por 365 


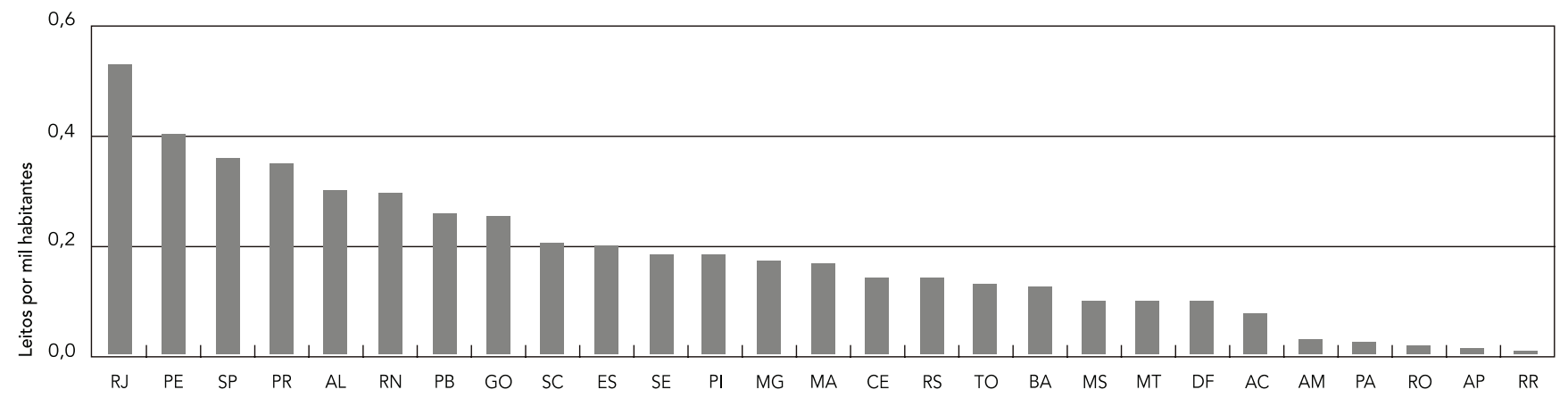

1b) Percentual de leitos psiquiátricos no total de leitos ocupados/Sistema Único de Saúde (SUS) por mil habitantes.

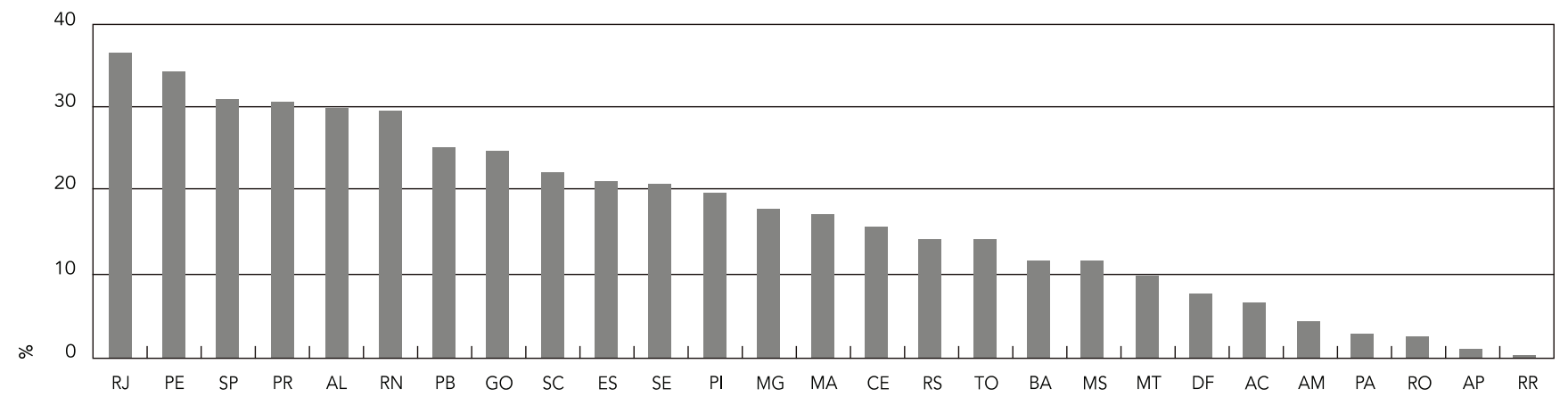

Fonte: Departamento de Informática do SUS 6,11.

profissionais e $\mathrm{R} \$ 74,37$ para SADT, totalizando R\$ 898,57 (Tabela 3).

O Ministério da Saúde estima que 15 mil pacientes asilares são potencialmente beneficiários de desinstitucionalização 5 . Os recursos a serem gerados com a desativação destes 15 mil leitos asilares são equivalentes a R\$ 162 milhões ao ano (a preços de 2004).

De acordo com a Tabela $4,78,8 \%$ do total de leitos psiquiátricos ocupados pelo SUS em 2004 eram privados. Entre os 67 hospitais com mais de 200 leitos psiquiátricos ocupados, 55 eram privados, 11 estaduais e um municipal. Os hospitais com mais de 200 leitos ocuparam $51,1 \%$ dos 45.194 leitos psiquiátricos e receberam $\mathrm{R} \$ 231$ milhões do SUS por serviços hospitalares, profissionais e SADT em 2004.

\section{Discussão}

A psiquiatria, em vez de tentar compreender as dimensões psicológicas da doença mental, concentra seus esforços na descoberta de causas orgânicas para todas as perturbações mentais. O diagnóstico psiquiátrico, apesar do empenho dos psiquiatras em estabelecer sistemas objetivos que lhes permitam incluir a doença mental na definição biomédica de doença, é, no entanto, notoriamente subjetivo 12 .

O pouco conhecimento das origens das doenças mentais e o diagnóstico "subjetivo", realizado a partir de síndromes, sinais e sintomas, são comumente utilizados para considerar o diagnóstico psiquiátrico como insuficiente na previsão da reabilitação do paciente ${ }^{13}$. Mas a Reforma Psiquiátrica trouxe o conceito de cidadania às discussões, admitindo "um novo lugar social para o sofrimento mental" 14 (p. 196). 
Leitos psiquiátricos ocupados/Sistema Único de Saúde (SUS) por especialidade e data de internação. Brasil, 2004.

\begin{tabular}{|c|c|c|c|c|c|c|c|}
\hline \multirow[t]{2}{*}{ Transtornos mentais e comportamentais } & \multirow[t]{2}{*}{ CID-10 } & \multicolumn{6}{|c|}{ Média anual * } \\
\hline & & \multicolumn{2}{|r|}{$\mathbf{n}$} & \multicolumn{2}{|c|}{$\%$} & \multicolumn{2}{|c|}{ Por mil habitantes } \\
\hline Orgânicos (demência etc.) & F00-09 & \multicolumn{2}{|r|}{6.292} & \multicolumn{2}{|c|}{13,9} & \multicolumn{2}{|c|}{0,04} \\
\hline Uso de substâncias psicoativas (álcool etc.) & F10-19 & \multicolumn{2}{|r|}{6.644} & \multicolumn{2}{|c|}{14,7} & \multicolumn{2}{|c|}{0,04} \\
\hline Esquizofrenia & F20-29 & \multicolumn{2}{|r|}{24.606} & \multicolumn{2}{|c|}{54,4} & \multicolumn{2}{|c|}{0,14} \\
\hline Depressão & F30-39 & \multicolumn{2}{|r|}{3.604} & \multicolumn{2}{|c|}{8,0} & \multicolumn{2}{|c|}{0,02} \\
\hline Retardo mental & F70-79 & \multicolumn{2}{|r|}{3.560} & \multicolumn{2}{|c|}{7,9} & \multicolumn{2}{|c|}{0,02} \\
\hline Outros & - & \multicolumn{2}{|r|}{489} & \multicolumn{2}{|c|}{1,1} & \multicolumn{2}{|c|}{0,00} \\
\hline Total & F00-99 & \multicolumn{2}{|r|}{45.194} & \multicolumn{2}{|c|}{100,0} & \multicolumn{2}{|c|}{0,25} \\
\hline \multirow[t]{3}{*}{ Transtornos mentais e comportamentais } & CID-10 & \multicolumn{6}{|c|}{ Média de dezembro por data de internação ** } \\
\hline & & \multicolumn{2}{|c|}{ Até junho de 2004} & \multicolumn{2}{|c|}{ Julho-dezembro de 2004} & \multicolumn{2}{|c|}{ Total } \\
\hline & & $\mathrm{n}$ & $\%$ & n & $\%$ & $\mathbf{n}$ & $\%$ \\
\hline Orgânicos (demência etc.) & F00-09 & 3.939 & 66,9 & 1.953 & 33,1 & 5.893 & 100,0 \\
\hline Uso de substâncias psicoativas (álcool etc.) & F10-19 & 416 & 6,5 & 5.962 & 93,5 & 6.378 & 100,0 \\
\hline Esquizofrenia & F20-29 & 10.260 & 43,3 & 13.443 & 56,7 & 23.703 & 100,0 \\
\hline Depressão & F30-39 & 270 & 7,5 & 3.309 & 92,5 & 3.578 & 100,0 \\
\hline Retardo mental & F70-79 & 2.883 & 82,6 & 608 & 17,4 & 3.491 & 100,0 \\
\hline Outros & - & 116 & 23,8 & 373 & 76,2 & 489 & 100,0 \\
\hline Total & F00-99 & 17.885 & 41,1 & 25.648 & 58,9 & 43.533 & 100,0 \\
\hline
\end{tabular}

CID-10: Classificação Internacional de Doenças, 10ạ Revisão 10.

Fonte: Ministério da Saúde?.

* Leitos ocupados (média anual): dias de permanência divididos por 365;

** Média em dezembro de 2004: dias de permanência divididos por 31.

O número de leitos psiquiátricos por habitante nos países ricos é muito superior à média verificada nas regiões mais pobres do planeta 15. Este fato pode sugerir tanto que os países mais desenvolvidos possuem uma maior rede de apoio institucional para pacientes com transtornos mentais, como pode sugerir que as sociedades economicamente menos desenvolvidas geram menos pacientes ou convivem melhor com as pessoas que apresentam transtornos mentais. Entre os países desenvolvidos, vale registrar, a Itália e a Espanha destacam-se pelo seu relativo baixo número de leitos por habitante 16 .

A discrepância entre o número de leitos psiquiátricos por habitante nas unidades da federação brasileira em 2004, expressa na Figura 1, da mesma forma, pode tanto significar desassistência nas regiões com menor número de leitos psiquiátricos por habitante, como saúde mental e inclusão social das pessoas que apresentam transtornos mentais. O fato de os estados com maiores taxas de leitos psiquiátricos ocupados por mil habitantes apresentarem maiores percentuais de leitos psiquiátricos no total de leitos ocupados pelo SUS parece confirmar a segunda alternativa (saúde mental e inclusão social). Lancman 17 considera também que os hospitais psiquiátricos, além de responsáveis pela cronificação dos doentes mentais, geram aumento de demanda aos serviços de saúde mental.

O número de leitos psiquiátricos ocupados estimado neste trabalho é consistente com o número de leitos registrado pelo CNES 8 , a diferença sendo justificada pelo número de leitos em manutenção. $\mathrm{O}$ número de leitos psiquiátricos cadastrados no SIH 7 é sistematicamente superior ao número de leitos registrados pelo CNES 8 .

A Reforma Psiquiátrica tem como objetivo a desinstitucionalização de pacientes psiquiátricos e o desenvolvimento de uma rede extrahospitalar capaz de garantir a reinserção social, a cidadania e o direito da pessoa portadora de transtornos mentais de atingir seu potencial de autonomia na comunidade 18,19 .

De acordo com a legislação em vigor no Brasil, os recursos financeiros gerados pela desativação dos leitos psiquiátricos devem ser necessariamente direcionados para a rede de serviços extra-hospitalares de saúde mental 20 . Há que se 
Valor pago/Sistema Único de Saúde (SUS) por leito psiquiátrico. Brasil, 2004.

\begin{tabular}{|c|c|c|c|c|c|}
\hline \multirow[t]{3}{*}{ Transtornos mentais e comportamentais } & \multirow[t]{3}{*}{ CID-10 } & \multicolumn{4}{|c|}{ Valor por paciente/mês (R\$) * } \\
\hline & & Serviços & Serviços & SADT & Total \\
\hline & & hospitalares & \multicolumn{3}{|l|}{ profissionais } \\
\hline Orgânicos (demência etc.) & F00-09 & 766,48 & 77,71 & 79,20 & 923,39 \\
\hline Uso de substâncias psicoativas (álcool etc.) & F10-19 & 769,99 & 77,08 & 74,30 & 921,38 \\
\hline Esquizofrenia & F20-29 & 746,86 & 74,50 & 73,76 & 895,13 \\
\hline Depressão & F30-39 & 750,47 & 72,95 & 71,58 & 895,00 \\
\hline Retardo mental & F70-79 & 695,54 & 73,04 & 73,39 & 841,96 \\
\hline Outros & - & 735,73 & 74,02 & 71,21 & 880,98 \\
\hline Total & F00-99 & 749,12 & 75,08 & 74,37 & 898,57 \\
\hline \multirow[t]{3}{*}{ Transtornos mentais e comportamentais } & \multirow[t]{3}{*}{ CID-10 } & \multicolumn{4}{|c|}{ Valor anual pago (R\$ mil) } \\
\hline & & Serviços & Serviços & SADT & Total \\
\hline & & hospitalares & profissionais & & \\
\hline Orgânicos (demência etc.) & F00-09 & 57.870 & 5.867 & 5.980 & 69.717 \\
\hline Uso de substâncias psicoativas (álcool etc.) & F10-19 & 61.387 & 6.145 & 5.924 & 73.456 \\
\hline Esquizofrenia & F20-29 & 220.527 & 21.999 & 21.779 & 264.305 \\
\hline Depressão & F30-39 & 32.453 & 3.154 & 3.095 & 38.702 \\
\hline Retardo mental & F70-79 & 29.715 & 3.120 & 3.135 & 35.970 \\
\hline Outros & - & 4.315 & 434 & 418 & 5.167 \\
\hline Total & F00-99 & 406.266 & 40.720 & 40.331 & 487.318 \\
\hline AlH: 15 mil leitos (R\$ mil) ** & F00-99 & 134.841 & 13.515 & 13.386 & 161.743 \\
\hline
\end{tabular}

CID-10: Classificação Internacional de Doenças, 10ạ Revisão 10; SADT: serviços auxiliares de diagnose e terapia.

Fonte: Ministério da Saúde 9.

* Valor anual pago dividido por dias de permanência no ano, multiplicado por 365/12;

** Recursos a serem gerados, ao ano, com a desativação de 15 mil leitos de pacientes asilares potencialmente beneficiários de desinstitucionalização.

considerar, contudo, que a verba das internações hospitalares é federal; a manutenção das residências terapêuticas e dos CAPS é municipal; e os hospitais psiquiátricos são essencialmente privados. Além disso, os internos nos hospitais psiquiátricos não são necessariamente originais dos municípios-sede dos hospitais em que estão internados, nem necessariamente permanecerão nestes municípios. Embora os recursos financeiros resultantes da desativação dos leitos psiquiátricos, de acordo com a legislação, devam ser realocados nos municípios de destino dos pacientes desinstitucionalizados 21 , não há garantia de que os recursos gerados pela diminuição de leitos na saúde mental sejam direcionados para o financiamento dos serviços extra-hospitalares, em especial quando o paciente retorna a um município diverso daquele em que estava hospitalizado 5.

Ainda que a desativação dos estimados 15 mil leitos psiquiátricos de pacientes asilares potencialmente beneficiários de desinstituciona- lização possa gerar anualmente R $\$ 162$ milhões passíveis de serem realocados para serviços psiquiátricos extra-hospitalares, o planejamento e a execução da Reforma Psiquiátrica têm sido muito tímidos. Furtado 22 destaca que as questões relativas ao financiamento representam o maior empecilho para a expansão dos SRT, e enfatiza a problemática do acesso e emprego dos recursos existente.

Em 2004, estavam operando no Brasil apenas 546 CAPS 5 e, em janeiro de 2005, o número de beneficiários do Programa De Volta para Casa atingia 952 dos estimados 15 mil potenciais beneficiários 5 . Considere-se ainda que o valor mensal do benefício do Programa De Volta para Casa, $\mathrm{R} \$ 240,00$, equivale a $32 \%$ do valor pago pelo SUS para os serviços hospitalares por leito psiquiátrico ao mês em 2004, R\$ 749,12 (líquidos de serviços profissionais e SADT).

Os hospitais privados receberam $\mathrm{R} \$ 388 \mathrm{mi}$ lhões do SUS em 2004 por 35.592 leitos psiquiátricos. Entre os hospitais psiquiátricos privados, 
Número de leitos psiquiátricos ocupados/Sistema Único de Saúde (SUS) por hospital. Brasil, 2004.

\begin{tabular}{|c|c|c|c|c|c|c|c|c|c|c|}
\hline \multirow[t]{2}{*}{ Número de leitos por hospital } & \multicolumn{2}{|c|}{ Até 40} & \multicolumn{2}{|c|}{$41-120$} & \multicolumn{2}{|c|}{$121-200$} & \multicolumn{2}{|c|}{ Mais de 200} & \multicolumn{2}{|c|}{ Total } \\
\hline & $\mathbf{n}$ & $\%$ & $\mathrm{n}$ & $\%$ & $\mathrm{n}$ & $\%$ & $\mathrm{n}$ & $\%$ & $\mathrm{n}$ & $\%$ \\
\hline Número de leitos ocupados * & 2.774 & 6,1 & 7.146 & 15,8 & 12.193 & 27,0 & 23.082 & 51,1 & 45.194 & 100,0 \\
\hline Privados & 1.670 & 3,7 & 5.270 & 11,7 & 10.287 & 22,8 & 18.365 & 40,6 & 35.592 & 78,8 \\
\hline Federais & 131 & 0,3 & 0 & 0,0 & 183 & 0,4 & 0 & 0,0 & 314 & 0,7 \\
\hline Estaduais & 574 & 1,3 & 1.593 & 3,5 & 1.513 & 3,3 & 3.989 & 8,8 & 7.670 & 17,0 \\
\hline Municipais & 398 & 0,9 & 283 & 0,6 & 210 & 0,5 & 727 & 1,6 & 1.617 & 3,6 \\
\hline Número de hospitais & 259 & 53,0 & 86 & 17,6 & 77 & 15,7 & 67 & 13,7 & 489 & 100,0 \\
\hline Privados & 153 & 31,3 & 62 & 12,7 & 65 & 13,3 & 55 & 11,2 & 335 & 68,5 \\
\hline Federais & 10 & 2,0 & 0 & 0,0 & 1 & 0,2 & 0 & 0,0 & 11 & 2,2 \\
\hline Estaduais & 54 & 11,0 & 20 & 4,1 & 9 & 1,8 & 11 & 2,2 & 94 & 19,2 \\
\hline Municipais & 42 & 8,6 & 4 & 0,8 & 2 & 0,4 & 1 & 0,2 & 49 & 10,0 \\
\hline Valor pago/SUS (R\$ mil) & 32.642 & 6,7 & 85.681 & 17,6 & 138.359 & 28,4 & 230.635 & 47,3 & 487.318 & 100,0 \\
\hline Privados & 20.247 & 4,2 & 65.082 & 13,4 & 117.459 & 24,1 & 184.762 & 37,9 & 387.551 & 79,5 \\
\hline Federais & 1.550 & 0,3 & 0 & 0,0 & 1.646 & 0,3 & 0 & 0,0 & 3.196 & 0,7 \\
\hline Estaduais & 6.740 & 1,4 & 17.089 & 3,5 & 17.019 & 3,5 & 38.882 & 8,0 & 79.729 & 16,4 \\
\hline Municipais & 4.105 & 0,8 & 3.510 & 0,7 & 2.235 & 0,5 & 6.991 & 1,4 & 16.841 & 3,5 \\
\hline
\end{tabular}

Fonte: Ministério da Saúde 9 .

Nota: todos os percentuais foram calculados em relação aos totais (de leitos, hospitais e valor pago/SUS).

* Leitos ocupados (média anual): dias de permanência divididos por 365 (dados consolidados para a Santa Casa de Nova Granada, Estado de São Paulo, que alterou a razão social durante o exercício de 2004).

55 ocupavam mais de 200 leitos e receberam $\mathrm{R} \$$ 185 milhões (média anual de $\mathrm{R}$ 3,4 milhões por hospital). A relação de todos os hospitais que recebem verba do SUS, assim como a série de variáveis relacionadas às AIH pagas, está disponível para tabulação nos CD-ROM distribuídos pelo Ministério da Saúde ${ }^{9}$.

O processo de desinstitucionalização dos pacientes psiquiátricos, evidentemente, afeta inúmeros interesses. Até a falta de certidão de nascimento de alguns internos tem sido muitas vezes apontada como empecilho à desinstitucionalização, apesar do registro de nascimento ser um direito do cidadão e sua emissão tardia poder ser agilizada pelo Ministério Público 5 .

Mas, significativo percentual de internos tem condições de residir fora do hospital, e mesmo os pacientes mais graves precisam de projetos terapêuticos específicos e não necessariamente de internação. Em geral, as instituições hospitalares brasileiras não possuem programas específicos dirigidos à reabilitação psicossocial 13 .

A precária rede extra-hospitalar tem sido utilizada como impedimento à desativação dos leitos psiquiátricos, embora esta gere os recursos necessários para a ampliação daquela. O responsável pela área da saúde mental da Prefeitura de São Paulo, o município mais abastado do país, declarou em 2004 que a cidade não tinha capacidade para suportar uma redução de leitos psiquiátricos e impediu o fechamento de uma clínica recomendado pelo Ministério da Saúde 23 .

Por fim, há que se considerar que a desinstitucionalização dos pacientes psiquiátricos, que tem como objetivo direto garantir a reinserção social, a cidadania e o direito da pessoa portadora de transtornos mentais de atingir seu potencial de autonomia na comunidade, deverá também romper o estigma contra os "loucos" e libertar os "normais" de seu aprisionamento à estrutura funcional das sociedades ditas modernas.

Nas sociedades modernas, por influência de Descartes, o corpo é visto como uma complexa máquina e a razão, privilégio do homem, estaria pairando muito acima do corpo. Eventuais descarrilamentos da máquina cerebral dos trilhos da razão, segundo esta concepção, devem ser devidamente consertados, por bem, ou por mal 2 .

“'Veja', dizia Ochwiay Biano [chefe dos índios Taos do Novo México] como os brancos têm um ar cruel. Tem lábios finos, nariz em ponta, os rostos sulcados de rugas e deformados. Os olhos têm uma expressão fixa, estão sempre buscando algo. O que procuram? Os brancos sempre desejam alguma coisa, estão sempre inquietos, e não conhecem o repouso. Nós não sabemos o que eles querem. Não 
os compreendemos e achamos que são loucos!".

"Perguntei-lhe [Jung] então por que pensava que todos os brancos eram loucos. Respondeu-me: 'Eles dizem que pensam com suas cabeças".

\section{Resumo}

O objetivo do trabalho é estimar o número de leitos psiquiátricos ocupados por Unidade da Federação e o valor pago pelo Sistema Único de Saúde (SUS) nas internações por serviços hospitalares, serviços profissionais, exames e medicamentos no Brasil em 2004. O número médio de leitos psiquiátricos ocupados, estimado a partir do total de dias de permanência no ano, e o valor pago pelo SUS foram obtidos a partir das Autorizações de Internação Hospitalar (AIH). O número de leitos psiquiátricos ocupados pelo SUS era de 45 mil em 2004. O valor total pago pelo SUS para internações de pacientes com transtornos mentais atingiu $R \$ 487$ milhões em 2004. Os hospitais privados eram responsáveis por $78,8 \%$ do total de leitos psiquiátricos ocupados pelo SUS. Ainda que a desativação de estimados 15 mil leitos asilares possa gerar anualmente $R \$ 162$ milhões ao ano passíveis de serem realocados para serviços psiquiátricos extra-hospitalares, o planejamento e a execução da Reforma Psiquiátrica têm sido muito tímidos. A precária rede extra-hospitalar tem sido utilizada como impedimento à desativação dos leitos psiquiátricos, embora esta gere os recursos necessários para a ampliação daquela.

Número de Leitos em Hospital; Hospitais Psiquiátricos; Transtornos Mentais
"Mas naturalmente! Com o que pensa você? - perguntei admirado".

“Nós pensamos aqui - disse ele, indicando o coração” 24 (p. 219).

\section{Colaboradores}

Todos os autores participaram de todas as etapas da pesquisa e elaboração do artigo.

\section{Agradecimentos}

À Maria Luiza Andrade Simões (psicóloga), Dagomar Heriques Lima (Tribunal de Contas da União), pela colaboração e aos pareceristas dos Cadernos de Saúde Pública, pelas sugestões. Este trabalho foi realizado com o apoio do Conselho Nacional de Desenvolvimento Científico e Tecnológico (CNPq). 


\section{Referências}

1. Crepet P, De Plato G. Psychiatry without asylums: origins and prospects in Italy. Int J Health Serv 1983; 13:119-29.

2. Silveira N. O mundo das imagens. São Paulo: Editora Ática; 1992.

3. Amarante P. Novos sujeitos, novos direitos: o debate em torno da reforma psiquiátrica. Cad Saúde Pública 1995; 11:491-4.

4. Presidência da República.Lein ${ }^{\circ} .10 .216$, de6 deabril de 2001. https://www.planalto.gov.br/ccivil_03/ leis/leis_2001/110216.htm (acessado em 19/Ago/ 2005).

5. Tribunal de Contas da União. Avaliação das ações de atenção à saúde mental: Programa Atenção à Saúde de Populações Estratégicas e em Situações Especiais de Agravos. Brasília: Tribunal de Contas da União; 2005.

6. Departamento de Informática do SUS. Morbidade hospitalar do SUS por local de internação - Brasil. http://tabnet.datasus.gov.br/cgi/deftohtm. exe?sih/cnv/miuf.def (acessado em 06/Set/2005).

7. Departamento de Informática do SUS. Rede hospitalar do SUS - Brasil. http://tabnet.datasus.gov. $\mathrm{br} / \mathrm{cgi} /$ deftohtm.exe?sih/cnv/cxuf.def (acessado em 18/Ago/2005).

8. Cadastro Nacional de Estabelecimentos de Saúde. Indicadores: leitos. http://cnes.datasus.gov. br/Mod_Ind_Tipo_Leito.asp (acessado em 18/ Ago/2005).

9. Ministério da Saúde. AIH: movimento de autorização de internação hospitalar - 2004 [CD-ROM] Brasília: Ministério da Saúde; 2005.

10. Organização Mundial da Saúde. Classificação estatística internacional de doenças e problemas relacionados à saúde, 10a revisão. São Paulo: Centro Colaborador da OMS para a Classificação de Doenças em Português; 1995.

11. Departamento de Informática do SUS. População residente - Brasil. http://tabnet.datasus.gov.br/ cgi/deftohtm.exe?ibge/cnv/popuf.def (acessado em 18/Ago/2005).
12. Capra F. O ponto de mutação. São Paulo: Editora Cultrix; 2002.

13. Gonçalves S, Fagundes P, Lovisi G, Lima LA. Avaliação das limitações no comportamento social em pacientes psiquiátricos de longa permanência. Ciênc Saúde Coletiva 2001; 6:105-13.

14. Oliveira AGB, Alessi NP. Cidadania: instrumento e finalidade do processo de trabalho na reforma psiquiátrica. Ciênc Saúde Coletiva 2005; 10:191-203.

15. World Health Organization. Atlas: mental health resources in the world 2001. Geneva: World Health Organization; 2001.

16. Salvador-Carulla L, Tibaldi G, Johnson S, Scala E, Romero C, Munizza C, et al. Patterns of mental health service utilisation in Italy and Spain: an investigation using the European Service Mapping Schedule. Soc Psychiatry Psychiatr Epidemiol 2005; 40:149-59.

17. Lancman S. Instituições psiquiátricas e comunidades: um estudo de demanda em saúde mental no Estado de São Paulo, Brasil. Cad Saúde Pública 1997; 13:93-102.

18. Eastman N. Mental health law: civil liberties and the principle of reciprocity. BMJ 1994; 308:43-5.

19. Bertolote JM. Legislação relativa à saúde mental: revisão de algumas experiências internacionais. Rev Saúde Pública 1995; 29:152-6.

20. Ministério da Saúde. Residências terapêuticas: o que são, para que servem. Brasília: Ministério da Saúde; 2004.

21. Ministério da Saúde. Portaria no. 52/GM de 20 de janeiro de 2004. http://www.sespa.pa.gov.br/Sus/ Portarias/PT2004/portaria0052.htm (acessado em 19/Ago/2005)

22. Furtado JP. Avaliação da situação atual dos serviços residenciais terapêuticos no SUS. Ciênc Saúde Coletiva 2006; 11:785-95.

23. Prefeitura diz que SP não tem como absorver mudança. Folha de S. Paulo 2004; 17 ago.

24. Jung CG. Memórias, sonhos, reflexões. Rio de Janeiro: Editora Nova Fronteira; 1995.

Recebido em 11/Jun/2007

Versão final reapresentada em 12/Fev/2008

Aprovado em 03/Mar/2008 